\title{
A Heart Surgery Simulator With an Integrated Supervision System for Self-Learning the Key Steps and Pitfalls of the Mirral Valve Repair
}

\author{
Initial Investigation
}

\author{
Piergiorgio Tozzi, MD; \\ Alessandra Solida, MD; \\ Giuseppe Siniscalchi, MD; \\ Enrico Ferrari, MD
}

Introduction: Over the years, surgical education has dramatically improved and has be-
come increasingly innovative. Almost all educational programs in surgery now rely on so-
phisticated training boxes and simulators that enable surgical instruments to be handled
and surgical procedures to be trained in a safe environment. However, simulators need
constant feedback from supervising senior surgeons, who only have limited teaching time
available. We describe a cardiac surgery simulator with an integrated supervision system
for self-learning how to repair a mitral valve. Methods: We developed a mitral surgery simulator with integrated sensors to generate, record, and display quantitative data on trainee performance in relation with the mitral valve repair procedure. A team of experienced cardiac surgeons defined critical areas of the model and an algorithm to identify inconsistent movements, in terms of error types and out-of-bound actions. The device provided real-time feedback on the accuracy of the stitches placed. Four experienced cardiac surgeons and 3 advanced cardiac-surgery used the simulator and were asked to evaluate specific parameters of the system on a scale ranging from 1 to 10 .

Results: All surgeons completed a P2 resection, followed by implanting a 32-mm mitral ring. The simulator detected 2 stitches that were placed in dangerous zones and another stitch that was placed in an inappropriate position. Users scored the real tissue feeling and interactivity of the model 9.5/10.

Conclusions: This heart-surgery simulator offers a reallife model for learning about and training in mitral valve surgery, which could potentially replace the experienced surgeon's teaching role.

(Sim Healthcare 00:00-00, 2021)

Key Words: Surgical simulator, mitral valve repair, simulation training, surgical training, simulation.

\section{$S$} chestedge-based specialty. Thus, surgical training programs must teach technical skills and behavior simultaneously. Over the years, surgical education has dramatically improved and has become increasingly innovative. Medical students and residents in surgical disciplines now benefit from highly sophisticated training boxes and simulators, which enable surgical instruments to be handled and surgical procedures to be trained. These learning tools help trainees acquire either basic skills or learn more advanced surgical procedures in a risk-free environment before performing real surgeries. This also reduces the clinical impact of novices' steep learning curves, which addresses institutional concerns about clinical

From the Lausanne University Hospital (P.T., A.S.), CHUV, Lausanne; Armed Forces Staff (G.S.), Berne; and Cardiocentro Lugano (E.F.), Lugano, Switzerland.

Correspondence to: Piergiorgio Tozzi, MD, Cardiac Surgery, Centre Hospitalier Universitaire Vaudois, Rue du Bugnon 46, CH-1011 Lausanne, Switzerland (e-mail: Piergiorgio.tozzi@chuv.ch).

The authors declare no conflict of interest.

Supplemental digital content is available for this article. Direct URL citations appear in the printed text and are provided in the HTML and PDF versions of this article on the journal's Web site (www.simulationinhealthcare.com).

Copyright ( 2021 Society for Simulation in Healthcare DOI: $10.1097 /$ SIH.0000000000000590 performance. Many universities in the United States and Great Britain use simulator-based curricula in their surgical programs, particularly for cardiac surgery programs. ${ }^{1,2}$

Certain surgical training boxes are sufficiently sophisticated that they can discriminate between different levels of surgical dexterity, which may help future surgeons further choose their surgical specialties. ${ }^{3}$ However, simulator-based training needs constant feedback from supervising senior surgeons, who typically only have limited time available for teaching purposes. These supervisors generally prefer to directly supervise students in real-life environments using apprenticeship-style training. Therefore, there is a clear need for a new generation of simulators that provide immediate positive and negative feedback about surgical gestures, even in the absence of a supervising surgeon. When innovative, cutting edge simulators are becoming increasingly available, more time in the work day should be made available to formally integrate them into the training program. In the past, we trained on 2 commercially available mitral valve repair simulators: the first uses a hydrogel mitral valve (LifeLike BioTissue, MA), which is simple but very realistic in terms of the tissue-like feel of the leaflets and subvalvular apparatus. The second was based on a thoracic torso with endoscopic and robotic access and disposable silicone mitral valve apparatus, which features a 

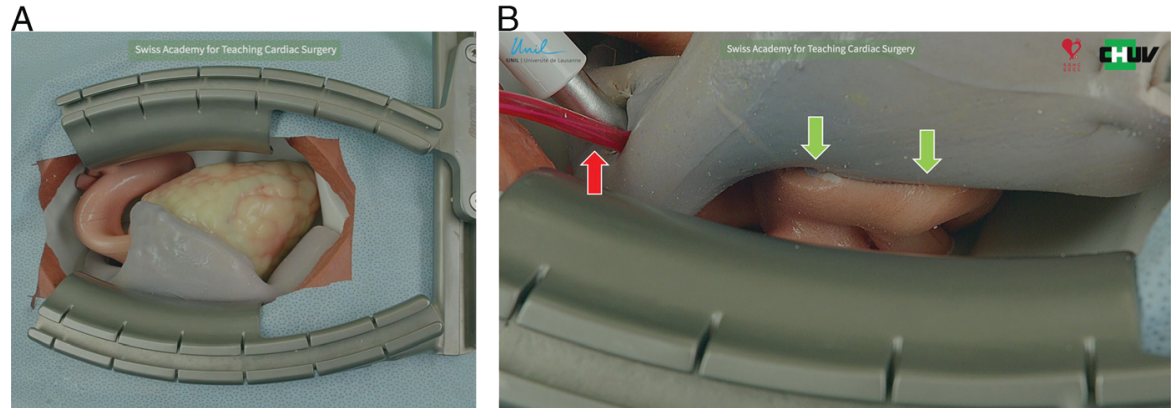

FIGURE 1. The model is a detailed reproduction of an adult human torso, using a 1:1 scale. It includes skin, subcutis, and bone tissues. The mediastinum hosts the silicone model of the heart, including the thoracic aorta, superior and inferior vena cava, and pulmonary arteries. The great vessels are patent and enable the positioning of cannulas for establishing extracorporeal circulation. Cannulas are secured using purse-string sutures. A, The sternum has been cut, and a Dubost retractor exposes the heart. B, Close-up view of the Waterstone groove (green arrows), where the surgeon opens the left atrium so as to expose the mitral valve. The red arrow indicates the cannula, which is positioned in the superior vena cava.

system that provides feedback on the accuracy of the procedure. This simulator has been extensively described in the literature. ${ }^{4}$ Based on our experience, we have developed a simulator that can also supervise a trainee during the mitral valve repair procedure, and we describe it in this article.

\section{METHODS}

We have developed the simulator that is depicted in Figure 1, with financial support from the School of Medicine at the Lausanne University, Switzerland, and the Swiss Society for Cardiac and Vascular Thoracic Surgery. We have also received technical support from a company that specializes in the development and production of surgical simulators and high-fidelity anatomy modeling (The Chamberlain Group, Great Barrington, MA). The device was designed to generate, record, and display quantitative data on trainee performance, in relation with the mitral valve repair procedure (Table 1 ). To design the geometry of the diseased mitral valve, we began by performing 3-dimensional reconstructions of computed tomographyderived DICOM images of patients with hearts exhibiting mitral valve regurgitation due to posterior leaflet prolapse, using dedicate software (Mimics Innovation Suite; Materialize NV, Leuven). Data were then transferred to CAD software (AutoCAD, V2015i) so as to refine the model. Hollow parts corresponded to vessels and cardiac chambers (Fig. 2). A cavity mold was then designed and fabricated to produce a life-sized synthetic heart using the silicone-molding technique. Five different types of silicone were used to mimic the specific feel of tissue from different anatomical parts of the heart (the atrium, ventricle, mitral valve leaflets, chordae tendineae, and aorta). Several sensors were embedded into the silicon mold. The breaking strength of the mitral leaflets was measured by passing a 5/0 polypropylene suture at the level of the insertion into the mitral annulus and pulling it radially with a dynamometer until disruption. A team of 4 experienced cardiac surgeons identified critical areas of the model. These concerned dedicated sensors that track surgical performance, as illustrated in Figure 3. The surgeons also defined an algorithm designed to identify inconsistent movements, in terms of error types and out-of-bound actions (Fig. 3). A micro camera was mounted onto an articulated arm, which recorded the entire procedure. Dedicated software processed video and sensor data in real time to reveal incorrect and dangerous stitches immediately on an interface with a 12 -in screen (Fig. 4). A 3-light witness display was also available for immediate feedback about the quality of surgical actions (Fig. 4). The surgical procedure was performed using standard surgical sutures and instruments, both for the full sternotomy and the minimally invasive approach. The system did not require the placement of electrodes on the surgical instruments. Four experienced cardiac surgeons and 3 advanced cardiac-surgery trainees performed a mitral annuloplasty using the simulator. Polypropylene (Prolene 2-0) and braided polyester (Ti-Cron 2-0) sutures were alternatively used to place a 32-mm Edwards Physio ring. The surgeons and trainees were then asked to evaluate specific parameters of the system on a scale ranging from 1 to 10 (Table 2). Data were expressed as median and interquartile range.

TABLE 1. Simulator Requirements and their Rationale

\begin{tabular}{ll}
\hline Requirement & $\begin{array}{l}\text { Specifications } \\
\text { Human-like appearance }\end{array}$ \\
$\begin{array}{l}\text { To simulate the entire surgical procedure for mitral valve repair/replacement, including cardiopulmonary bypass establishment } \\
\text { and surgical exposure of the valve using retractors. } \\
\text { Each part of the model should reproduce the texture of the real tissue. } \\
\text { Objective and real-time feedback on suture placement (appropriate/inappropriate) and possible lesions caused (out of the bounds/ } \\
\text { tissue damage). Possibility to review the procedure and the wrong steps. } \\
\text { Recording the time to complete the procedure. This function allows an expert supervision-free learning and training platform. }\end{array}$ \\
$\begin{array}{l}\text { The design of the model should be compatible with multiple surgical approaches (minimal invasive and full sternotomy). It can be } \\
\text { used several times and can be repaired. Ancillary components should last for minimum } 10 \text { procedures and be easily replaced. } \\
\text { Ther interface }\end{array}$ \\
$\begin{array}{l}\text { The user interface should clearly show the details of the procedure and pitfalls in real time. It is also compatible with multi users. } \\
\text { The size and weight have to be compatible with easy transport and storage. }\end{array}$
\end{tabular}




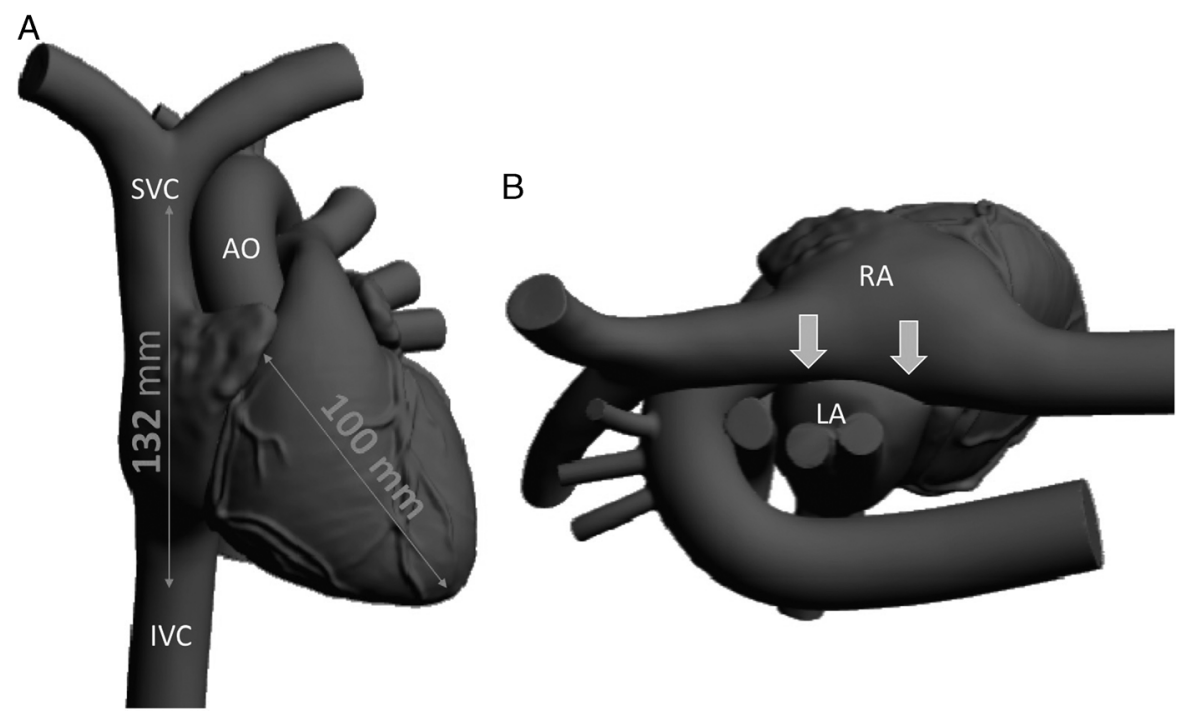

FIGURE 2. Heart design elaborated from computed tomography scan data of a human heart using CAD software. A, Antero-posterior view of the model; Ao, aorta; IVC, inferior vena cava; SVC, superior vena cava. Measures are expressed in millimeters. B, Right-side view of the model, which corresponds to the surgical view of the heart during minimally invasive mitral surgery. The green arrow indicates the Waterstone groove between the right atrium and left atrium.

Our ethical review committee (institutional review board) has reviewed and approved this study, and all participants gave their written consent to participate.

\section{RESULTS}

The thickness of the mitral valve leaflets' free edge was $0.8 \pm 0.2 \mathrm{~mm}$, and the thickness of the leaflets was $1.8 \pm 0.2 \mathrm{~mm}$ at the insertion into the mitral annulus. The transition between the maximum and minimum thickness was progressive and smooth. The breaking strength of the samples used in this study was between $12 \pm 0.4 \mathrm{MPa}$ (posterior leaflet) and $15 \pm 0.8 \mathrm{MPa}$ (anterior leaflet), which align with the values measured in humans. ${ }^{5}$

A full sternotomy approach (skin incision and sternum section with oscillating saw) was selected to test the simulator and validate the procedure. With the exception of a working heart-lung machine, all the steps of human procedure were simulated. A junior trainee placed cannulas for a cardiopulmonary bypass into both the vena cava and ascending aorta. Next, the trainees opened the left atrium in the Waterstone groove and exposed the mitral valve (Fig. 5). After valvular analysis, a P2 quadrangular resection was performed, which was followed by implanting a 32-mm Edwards Physio II ring (Fig. 6). Upon the implant procedure, the simulator detected 2 stitches that were placed in dangerous zones (Fig. 7) and another stitch that was placed in an inappropriate position (Fig. 7). We illustrated this feature of the simulator in a video (see video, Supplemental Digital Content 1, http://links.lww.com/SIH/A691, which demonstrates that the system displays incorrect stitches).

After completing the valvular repair, the test filled the left ventricle with water to confirm that the mitral valve was adequately repaired. The left atriotomy was closed, and the cannulas were retrieved. Results of the user's evaluation are detailed in Table 2 .

\section{DISCUSSION}

For several reasons, repairing a leaking mitral valve is undoubtedly one of the most complex cardiac-surgery procedures to
A

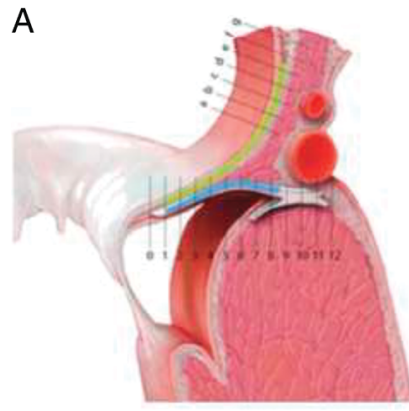

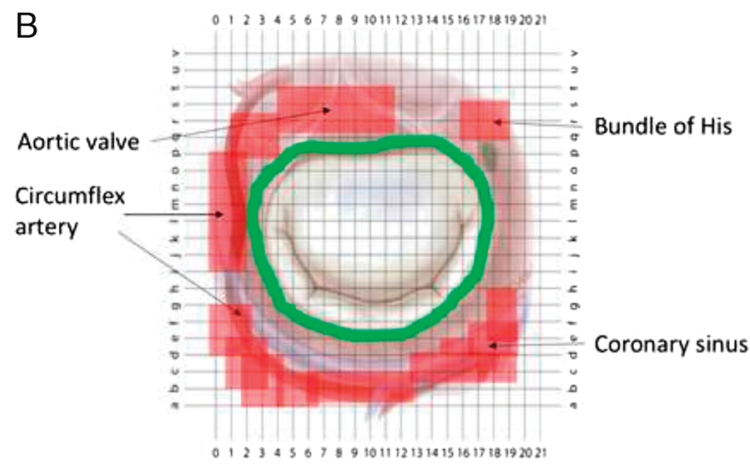

FIGURE 3. Mapping grid illustrating the sensors' layers and zoning, which assess the accuracy of a stitch. A, Cross-sectional view of the mapping grid. Green and red indicate the sensor layers that are made of conductive material, which are embedded in the silicone mold. Stitches 4-8 were considered perfect stitches; stitches 0-3 and 9-12 were considered incorrect stitches; A, shows a perfect stitch toward the anulus, until stitch 5; B to $G$, show incorrect stitches. B: Surgical view of the mitral valve. The green line corresponds to the correct area for placing the stitches. The red area corresponds to dangerous zones, in which the stitch could damage vital structures. Stitches placed outside the green and red areas are considered out-of-bound stitches. 


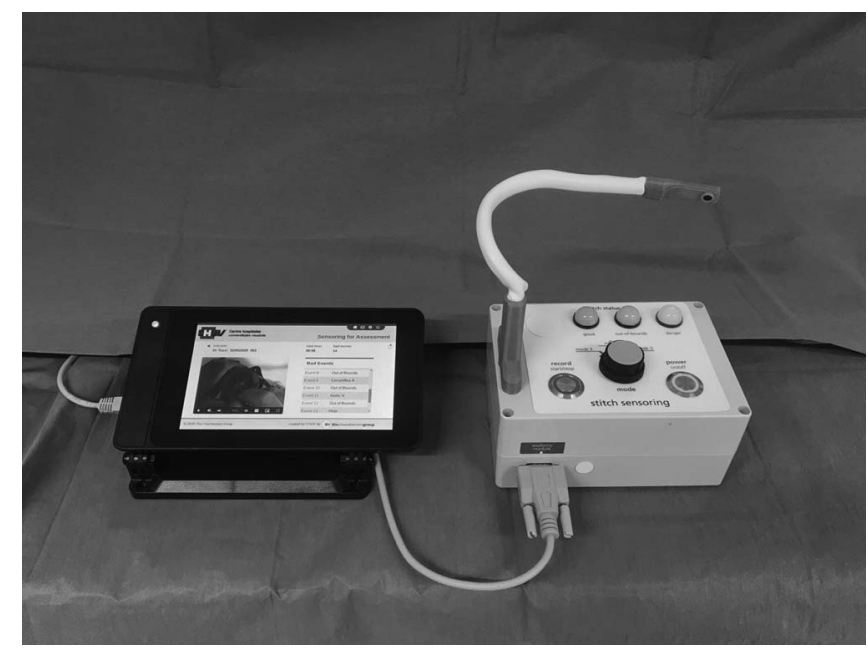

FIGURE 4. Overview of the supervision system, which comprises a user interface with a 12-in touch screen so as to review the procedure's recorded steps. This includes parameters that identify the trainee, date and time, and type or error (out of bounds, circumflex artery, coronary sinus, aortic valve, and conduction system). A control unit comprises an on-off button and a panel of lights to provide rapid feedback about the accuracy of the stitch when the trainee is passing it la red light indicates danger, a blue light is used for outof-bound stitches, and a green light reflects an appropriate stitch). A micro camera is mounted onto an articulated arm to film the surgical procedure.

both teach and learn. First, limited surgical exposure typically only enables one surgeon to clearly see the mitral valve at any one time. An assistant, who holds the sutures or suction/ retractor instruments, cannot clearly see what is happening. To present the procedure's key steps to the trainee, the surgeons must slow down his pace of repair and move their head away from the operating field. This is stressful and timeconsuming for all team members, and it can result in adverse effects on clinical outcomes, particularly for frail patients. This

TABLE 2. User's Simulator Evaluation (4 Experienced Cardiac Surgeons and Three Advanced Trainees in Cardiac Surgery)

\begin{tabular}{lr}
\hline Parameter & Score (out of 10), Median (IQR) \\
\hline General comments & \\
Human-like appearance & $9(1)$ \\
Consistent and reproducible procedure & $9(2)$ \\
Overall evaluation and reliability & $9(1)$ \\
Real tissue feeling & \\
Aorta & $9(2)$ \\
Right atrium & $9(1)$ \\
Left atrium & $9(1)$ \\
Surgical exposure & $10(0)$ \\
Mitral annulus & $9(1)$ \\
Mitral leaflets & $10(0)$ \\
Sutures placement & $10(0)$ \\
Sutures retention & $9(2)$ \\
Surgical procedure & \\
Realism of mitral valve prolapse & $9(2)$ \\
Realism of quadrangular resection & $10(0)$ \\
Realism of neocordae placement & $9(1)$ \\
Realism of mitral ring placement & $10(0)$ \\
Evaluation of the surgical repair & $9(2)$ \\
Interactivity & \\
Accuracy of suture placement information & $9(1)$ \\
Accuracy of potential lesions caused & $9(1)$ \\
Review process and clarity of feedback & $9(2)$ \\
Utility as surgical skills improvement tool & $9(1)$ \\
\hline IQR, interquartile range. &
\end{tabular}

renders the apprentice model in humans both unethical and contrary to institutional concerns about clinical performance. Moreover, surgical residents currently have significantly less time to devote to surgical training, because of work-time restrictions that have been introduced over the last 10 years. These limitations, in combination with increasing numbers of complex patient profiles, further restrict trainees' access to simple surgical procedures. For these reasons, most surgeons and students are not comfortable with this approach to teaching and subsequently welcome the introduction of simulators in this field.

In cardiothoracic surgery, simulation has been proposed for trainees as an approach to advance their proficiency of skills and for board-certified cardiac surgeons to become skilled at new techniques and challenging surgical procedures, including mitral valve surgery. ${ }^{6}$

Joyce et $\mathrm{al}^{7}$ reported that training simulators for learning complex surgical procedures have been developed to teach the knowledge and skills required for surgeons to be confidently self-managed during cardiac surgery. ${ }^{8-10}$

However, there are 2 major concerns about the effectiveness of simulators, which concern the system's ability to help learners achieve the learning objectives and whether the skills that are acquired can be transferred to patients. ${ }^{11,12}$ Both of these concerns could be addressed by creating lifelike models. The simulator presented in this study reproduces all the details of human anatomy, such as the texture of different tissues and the feeling created by passing a needle through them, the bleeding caused by lesions on the great vessels, and the possibility of reproducing all the technical steps of the mitral repair procedure.

This simulator represents a new generation of interactive surgical simulators that can be used for standardized learning. This could address both of the major concerns that were previously discussed, and it could also help achieve learning objectives, even in the absence of a teacher. A heart simulator that has an integrated supervision system (ISS) enables the 

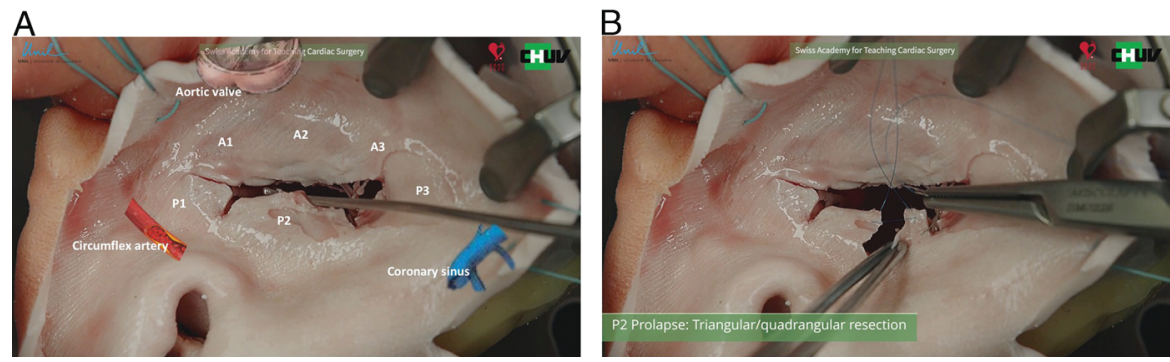

FIGURE 5. The left atrium has been opened, and the mitral valve has been exposed by the Cosgrove retractor. A, The mitral valve is inspected, and a P2 prolapse is identified. Overlay drawings illustrate the position of anatomical structures that must be recognized upon placing the stitches. B, The P2 segment has been resected, and the repair has been initiated using $5 / 0$ interrupted sutures.

trainee to practice the mitral repair without constant feedback from the teacher. The ISS reproduces the apprenticeship training model, in which the machine replaces experienced surgeons by telling the user when a stitch has been placed in an incorrect position, as well as the type of lesion that this incorrect stitch could have caused. These incorrect gestures can be reviewed as many times as necessary, and if there is doubt, the video can also be reviewed by an experienced surgeon for further comments or suggestions.

Another advantage of the simulator that is presented in this study is that it can be used several times, and it can even replicate a simulated case scenario. Each part of the heart, except for the mitral leaflets, can be repaired in a matter of minutes using a specifically developed repair tool kit. In the case of severe damage, individual parts can be independently replaced (the right atrium, vena cava, and aorta) because the different parts are magnetically connected. During the tests, all sensors worked properly for more than 10 full annuloplasty procedures using mitral ring placements.

The model's embedded tissue sensors more accurately evaluate suture placement, compared with motion-capture video or tracking. This represents the key element of an ISS simulator. In motion-capture sessions, images from multiple cameras are applied to calculate the 3-dimensional position of the targeted element, but sometimes, none of these cameras can gain a clear view. Sardari Nia et al ${ }^{4,9}$ developed a mitral valve simulator that acquires images from four cameras, with a resolution of 1.3 megapixels. To detect and measure the depth of sutures, they developed a software algorithm that could differentiate between new and already placed sutures by subtracting a reference image (in the background) and then calculating the depth of the new stitch in pixels, which is subsequently converted to millimeters. This system does not provide feedback on either the exact position of the suture, with respect to the mitral annulus, or on the lesion that could possibly be caused by the incorrect suture placement.

The full simulator, including its electronic components, can be stored in a small box that can be easily transported, which means that the trainee can freely practice mitral surgery and still receive feedback from the electronic supervisor.

The feedback received from the independent users, including experienced surgeons, was extremely positive, both in terms of the system's fidelity to reality and the consistency of the ISS-provided information. In particular, users appreciated the varying textures of different parts of the model, including the mitral valve itself, as well as the fact that the trainee must undertake all the required steps to properly expose the mitral valve, including the establishment of extracorporeal circulation, the left atriotomy, and the water test to check the valve's competence. Moreover, the model is compatible with both minimally invasive and open-chest approaches. The information provided by the ISS was clear, and it was delivered at the right time, which meant that the user had the opportunity to immediately see incorrect stitches and adjust them in compliance with instructions from the machine.

This simulator also constitutes a platform enabled to generate uniform skill assessments, demonstrate proficiency, and maintain certification standards within the field of mitral valve repair. It could also create a new means of remote dry-lab teaching, in which an experienced surgeon works with a trainee remotely, checks actions in real time or using recorded images, and assesses the quality of the surgical repair based on the ISS report.

This ISS heart-surgery simulator offers a lifelike model for learning about and training in mitral valve surgery, which could potentially replace the experienced surgeon's teaching
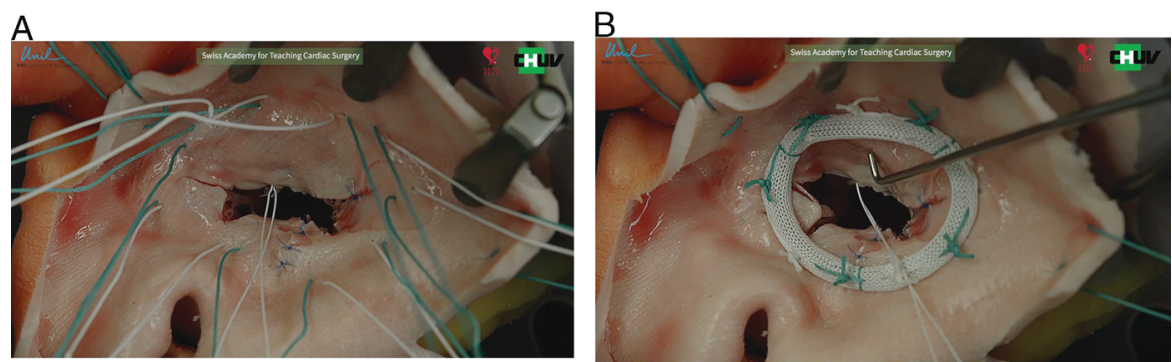

FIGURE 6. Quadrangular resection of the P2, followed by implantation of an Edwards Physio 32-mm ring. A, Sutures passed through the mitral annulus after restoring the integrity of the posterior leaflet. B, The annuloplasty ring has been placed, and neochordae on $A 2$ have been inserted to improve the leaflets' coaptation. 

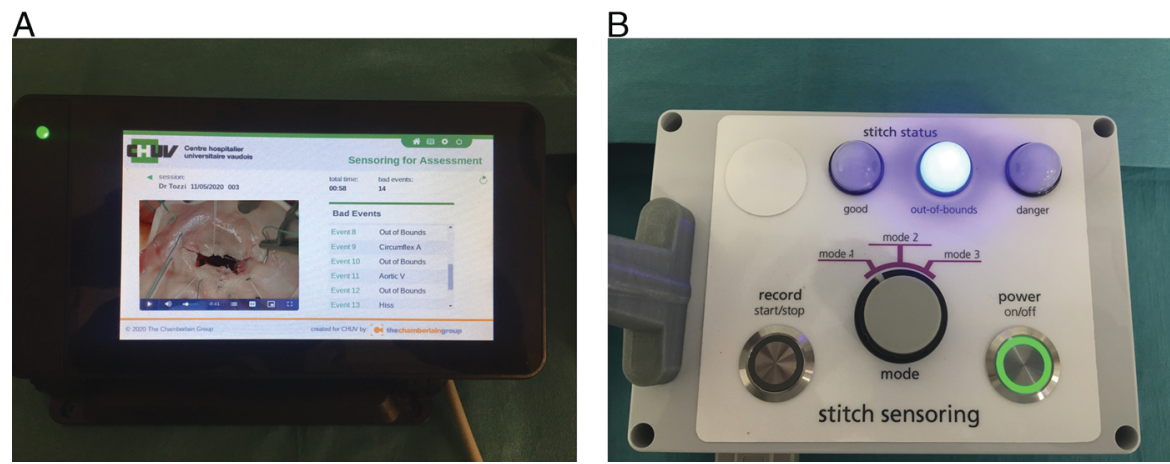

FIGURE 7. Feedback from the simulator upon placement of an inappropriate stitch. The stitch, which is at the P3 level, was considered out of bounds, with the blue light activated.

role. This system provides immediate and precise feedback about the accuracy of suture placement and is expected to be commercialized in 2022 at a competitive price.

\section{ACKNOWLEDGMENTS}

The authors thank the University of Lausanne, School of Medicine, and the Swiss Society for Cardiac and Vascular Thoracic Surgery for their strong support.

\section{REFERENCES}

1. Feins RH, Burkhart HM, Conte JV, et al. Simulation-based training in cardiac surgery. Ann Thorac Surg 2017;103:312-321.

2. Allum W. Improving surgical training. Surgery (Oxf) 2020;38(10): 596-600.

3. Zia A, Sharma Y, Bettadapura V, Sarin EL, Essa I. Video and accelerometer-based motion analysis for automated surgical skills assessment. Int J Comput Assist Radiol Surg 2018;13:443-455.

4. Sardari Nia P, Daemen JHT, Maessen JG. Development of a high-fidelity minimally invasive mitral valve surgery simulator. J Thorac Cardiovasc Surg 2019;157:1567-1574.

5. Hasan A, Ragaert K, Swieszkowski W, et al. Biomechanical properties of native and tissue engineered heart valve constructs. J Biomech 2014;47:1949-1963.
6. Holzhey DM, Seeburger J, Misfeld M, Borger MA, Mohr FW. Learning minimally invasive mitral valve surgery: a cumulative sum sequential probability analysis of 3895 operations from a single high-volume center. Circulation 2013;128:483-491.

7. Joyce DL, Dhillon TS, Caffarelli AD, et al. Simulation and skills training in mitral valve surgery. $J$ Thorac Cardiovasc Surg 2011;141:107-112.

8. Fann JI, Feins RH, Hicks GL, et al. Evaluation of simulation training in cardiothoracic surgery: the Senior Tour perspective. J Thorac Cardiovasc Surg 2012;143:264-272.

9. Sardari Nia P, Heuts S, Daemen JHT, Olsthoorn JR, Chitwood WR, Maessen JG. The EACTS simulation-based training course for endoscopic mitral valve repair: an air-pilot training concept in action. Interact Cardiovasc Thorac Surg 2020;30:691-698.

10. Joyce DL, Lahr BD, Maltais S, et al. Integration of simulation components enhances team training in cardiac surgery. $J$ Thorac Cardiovasc Surg 2018;155:2518-24.e5.

11. Heskin L, Simms C, Holland J, Traynor O, Galvin R. A systematic review of the educational effectiveness of simulation used in open surgery. Simul Healthe 2019;14:51-58.

12. Price J, Naik V, Boodhwani M, Brandys T, Hendry P, Lam BK. A randomized evaluation of simulation training on performance of vascular anastomosis on a high-fidelity in vivo model: the role of deliberate practice. J Thorac Cardiovasc Surg 2011;142:496-503. 